\title{
A NEW PROOF OF A CONJECTURE OF SCHILD
}

\author{
E. GRAY AND A. SCHILD
}

1. Introduction. In a paper, published in this Journal [3], one of the authors has introduced and discussed the class of functions $S_{p}$, having $|z|=1$ as radius of schlichtness and being of the form $f_{p}(z)$ $=z-\sum_{n=2}^{N} a_{n} z^{n}$, with the $a_{n}$ real and non-negative for $n=2,3, \cdots$, $N, N \geqq 2$. All functions of this class map the unit circle into starlike regions [3, Theorem 3$]$.

Let $d^{*}$ be the shortest distance from $w=0$ to $w=f_{p}\left(e^{i \theta}\right), 0 \leqq \theta<2 \pi$, and $d_{0}$ the shortest distance from $w=0$ to $w=f_{p}\left(r_{0} e^{i \theta}\right), 0 \leqq \theta<2 \pi$, where $r_{0}$ is the radius of convexity of $w=f_{p}(z)$. Among other things it was proved [3, Theorem 7] that for all $f_{p}(z) \in S_{p}$ we have $d_{0} / d^{*} \geqq 2 / 3$. It was conjectured there that for $f_{p}(z) \in S_{p}$ we actually have $d_{0} / d^{*}$ $\geqq 3 / 4$, attained by the function $f_{p}(z)=z-z^{2} / 2 \in S_{p}$.

The class of functions $S_{p}$ was discussed further and extended by Z. Lewandowski [1] and the truth of the conjecture $d_{0} / d^{*} \geqq 3 / 4$ for all $f_{p}(z) \in S_{p}$ was demonstrated by him in a second paper [2].

It is the aim of this short note to give an elementary and simple proof of the conjecture: $d_{0} / d^{*} \geqq 3 / 4$ for all $f_{p}(z) \in S_{p}$.

2. Proof of the conjecture that $d_{0} / d^{*} \geqq 3 / 4$ for all $f_{p}(z) \in S_{p \text {. The map }}$ of $|z|=r, 0<r \leqq 1$, by any $f_{p}(z) \in S_{p}$ will have its closest point from the origin on the positive real axis for $z=r$, since

$$
\left|f_{p}(z)\right|=\left|z-\sum_{n=2}^{N} a_{n} z^{n}\right| \geqq|z|-\sum_{n=2}^{N} a_{n}|z|^{n}=r-\sum_{n=2}^{N} a_{n} r^{n}=f_{p}(r) .
$$

We must show, therefore, that

$$
d_{0} / d^{*}=\left\{r_{0}-\sum_{n=2}^{N} a_{n}^{n} r_{0}^{n}\right\} /\left\{1-\sum_{n=2}^{N} a_{n}\right\} \geqq 3 / 4,
$$

where $r_{0}$ is the radius of convexity of $f_{p}(z)$.

$$
\begin{aligned}
d_{0} / d^{*}-3 / 4 & =\left\{r_{0}-\sum_{n=2}^{N} a_{n} r_{0}^{n}\right\} /\left\{1-\sum_{n=2}^{N} a_{n}\right\}-3 / 4 \\
& =\left\{r_{0}-3 / 4+\sum_{n=2}^{N} a_{n}\left[3 / 4-r_{0}^{n}\right]\right\} /\left\{1-\sum_{n=2}^{N} a_{n}\right\} .
\end{aligned}
$$

Since $\left\{1-\sum_{n-2}^{N} a_{n}\right\}>0[3$, Theorem 1], it is sufficient to show that $y=\left(r_{0}-3 / 4\right)+\sum_{n=2}^{N} a_{n}\left(3 / 4-r_{0}^{n}\right) \geqq 0$.

Received by the editors August 17, 1963. 
We recall [3, Theorem 5] that $r_{0}$ is the least positive root of $\sum_{n=2}^{N} n^{2} a_{n} r^{n-1}=1$ and, therefore, $\sum_{n=2}^{N} n^{2} a_{n} r_{0}^{n-1}=1$. The expression for $y$ can now be written in the form:

$$
\begin{aligned}
y & =\left(r_{0}-3 / 4\right) \sum_{n=2}^{N} n^{2} a_{n} r_{0}^{n-1}+\sum_{n=2}^{N} a_{n}\left(3 / 4-r_{0}^{n}\right) \\
& =\sum_{n=2}^{N} a_{n}\left\{n^{2} r_{0}^{n}-(3 / 4) n^{2} r_{0}^{n-1}+3 / 4-r_{0}^{n}\right\} .
\end{aligned}
$$

The proof will now be completed by showing that

$$
z(n)=\left\{n^{2} r_{0}^{n}-(3 / 4) n^{2} r_{0}^{n-1}+3 / 4-r_{0}^{n}\right\} \geqq 0 \text { for } n=2,3, \cdots, N .
$$

Clearly, $z(2)=3 r_{0}^{2}-3 r_{0}+3 / 4=3\left(r_{0}-1 / 2\right)^{2} \geqq 0$. For $n \geqq 2$, we consider

$$
\begin{aligned}
g(n) & =z(n+1)-z(n) \\
& =r_{0}^{n-1}\left\{\left(n^{2}+2 n\right) r_{0}^{2}-\left(7 n^{2} / 4+3 n / 2-1 / 4\right) r_{0}+3 n^{2} / 4\right\} .
\end{aligned}
$$

It was shown [4, Lemma 3.4] that $d_{0} / d^{*}>r_{0}$, for all functions $w=z+\sum_{n=2}^{N} a_{n} z^{n}$, regular, schlicht and starlike in the unit circle. This result will, therefore, also hold for $f_{p}(z) \in S_{p}$, and since, for this class of functions, $r_{0} \geqq 1 / 2[3$, Theorem 5$]$, it is sufficient to prove the conjecture for $1 / 2 \leqq r_{0}<3 / 4$. It is, therefore, convenient to set $r_{0}=3 / 4-x$, where $0<x \leqq 1 / 4$, in the expression for $g(n)$. For any particular $n$, the coefficient of $r_{0}^{n-1}$ in $g(n)$ becomes $h(x)=\left(n^{2}+2 n\right) x^{2}$ $+(1 / 4)\left(n^{2}-6 n-1\right) x+3 / 16$. It is clear that $h(x)>0$ for $n \geqq 6$. Also, the discriminant of $h(x)$ is $\Delta=(1 / 16)\left(n^{4}-12 n^{3}+22 n^{2}-12 n+1\right)$ $=(1 / 16)(n-1)^{2}\left(n^{2}-10 n+1\right)$. Obviously, $\Delta<0$ for $n<10$, and since $h(0)>0, h(x)>0$ for $n=2,3,4,5$ also. Therefore, $z(n+1)-z(n)>0$ for $n=2,3,4, \cdots$ and since $z(2) \geqq 0$, the conjecture is proved.

\section{REFERENCES}

1. Z. Lewandowski, Quelques remarques sur les theorems de Schild relatifs d une classe de fonctions univalentes, Ann. Univ. Mariae Curie-Sklodowska Sect. A (9) 9 (1955), 149-155.

2. - Nouvelles remarques sur les theorèmes de Schild relatifs d une classe de fonctions univalentes (Demonstration d'une hypothèse de Schild), Ann. Univ. Mariae Curie-Skłodowska Sect. A (8) 10 (1956), 81-94.

3. A. Schild, On a class of functions schlicht in the unit circle, Proc. Amer. Math. Soc. 5 (1954), 115-120.

4. - On a problem in conformal mapping of schlicht functions, Proc. Amer. Math. Soc. 4 (1953), 43-51.

\section{General Electric Company and TEMPLE UNIVERSITY}

